\title{
The Design of A Remote High Precision Temperature Acquisition System Based on GPRS
}

\author{
Hong $\mathrm{Xu}^{1}$, Fanrong Meng ${ }^{2,3, a}$,Xinhong Wang ${ }^{2,3}$, \\ Yonggang Qian ${ }^{2,3}$, Chuncheng Zhou ${ }^{2,3}$, Wei Li $^{2,3}$ \\ ${ }^{1}$ The High-Tech Research \& Development Center Ministry of Science \& Technology, \\ P,R,C, No. 9 Building XiYuan Hotel, No. 1 San Li He Road, Beijing, China \\ ${ }^{2}$ Key Laboratory of Quantitative Remote Sensing Information Technology, No. 9, Deng \\ Zhuang South Road HaiDian District, Beijing, China \\ ${ }^{3}$ Academy of Opto-Electronics, Chinese Academy of Sciences, No. 9, Deng Zhuang South \\ Road HaiDian District, Beijing, China \\ amengfanrong@aoe.ac.cn
}

\begin{abstract}
Keywords: High Precision, Pt100, GPRS, Remote
Abstract. Surface temperature is an important parameter commonly used in thermal infrared remote sensing. Precise measurement of temperature is one of basic tasks in ground parameter acquisition. A remote high precision temperature acquisition system based on GPRS is presented in this paper, which consists of a STM32 MCU acting as main controller, a temperature measurement module made up of PT100 sensor and AD7787, a wireless data transmission module using Neoway GPRS chip as core device, and auxiliary modules such as OLED display, Micro SD card memory, RS232 configuration interface. The paper describes in detail the Pt100 3-wire temperature measurement method, circuit design and software controlling of the system. Test on the system was done and results indicate that thermomeric error of this system is less than $0.2^{0} \mathrm{C}$. The system has function of remote data trasmission, so can easily realize unattended temperature collection in field applications, taking advantage of low-cost and labor-saving, and is expected to have good practical values.
\end{abstract}

\section{Introduction}

Field measurement plays an important role in various remote sensing campaigns. In thermal infrared (TIR) remote sensing, we usually need to perform accurate and continuous temperature measurements on the TIR targets. The traditional way which depends on manual measurement is cost-consuming and requires human resource supports, and it will inevitably introduce impact of subjective factor on the measurement. So it is obviously valuable to realize a system for unattended remote high precision temperature acquisition. With the development of wireless communication and IOT (internet of things) technologies, mobile signals become accessible nearly all over the world. A variety of maturely developed GPRS modules has been put into market, hence accessing internet through GPRS to realize remote data transmission has been a good practical choice for many automated applications. The temperature acquisition system proposed in this paper will use GPRS module to realize remote transmission of temperature measurement data, and it will greatly improve the automation level of field measurement to obtain high efficiency on continuous measurement. This design solution can easily be applied to various measurement fields and targets.

\section{System Design}

Fig. 1 shows the functional block diagram of the remote high precision temperature acquisition system based on GPRS. The STM32F103 ${ }^{[1]}$ series MCU was adopted in our system. This series of MCU uses ARM Cortex-M3 kernel, supports maximum operating frequency $72 \mathrm{MHz}$, integrates 
SRAM up to $96 \mathrm{~KB}$ and FLASH up to $1 \mathrm{MB}$, has different communication interfaces (e.g. 232, 485, SDIO, Can), and can operate in multiple low-power modes (e.g. Sleep, Stop, Standby). In support of the STM32F103 MCU, we designed the temperature data collecting circuit, the GPRS telecommunication module, the OLED display module, the SD card memory module and the RS232 configuration interface. The MCU uses SPI interface to control OLED display and ADC sampling, uses a RS232 interface to set parameters such as server address and ports, uses another RS232 interface to communication with the GPRS module to realize transmission of acquired data, uses Analog Switch to control multi-channel voltage signal collection, and uses SDIO interface to implement data IO with the Micro SD card.

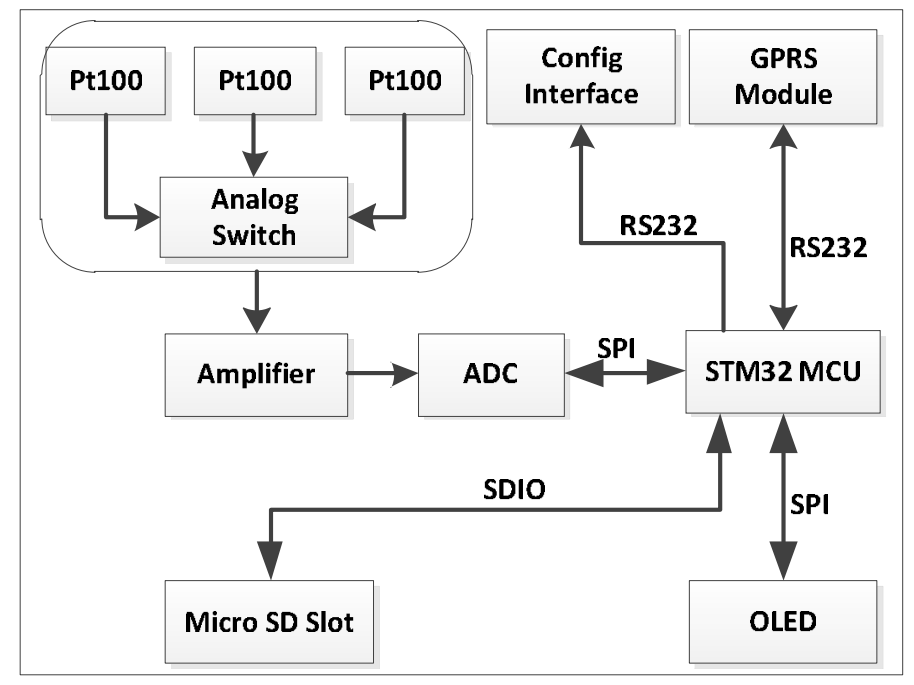

Fig. 1 Structure diagram of the proposed system

\section{High Precision Temperature Measurement Principles}

The Pt100 temperature sensor is a kind of resistive transducer made of metal Pt, which resistance value changes with temperature of the measured object, complying with the following relation:

$$
R= \begin{cases}R_{f}\left(1+A t+B t^{2}+a^{3}\right) & -200{ }^{\circ} \mathrm{C}<t<0{ }^{\circ} \mathrm{C} \\ R_{b}\left(1+A t+B t^{2}\right) & 0{ }^{\circ} \mathrm{C}<t<850{ }^{\circ} \mathrm{C} .\end{cases}
$$

Where Rt is Pt100's resistance value at temperature t ${ }^{0} \mathrm{C}, \mathrm{R}_{0}$ is Pt 100 's resistance value at temperature $0{ }^{0} \mathrm{C}, \mathrm{R}_{0}=100 \Omega$, and the coefficients $\mathrm{A}=3.968 \times 10^{-30} \mathrm{C}^{-1}, \mathrm{~B}=-5.847 \times 10^{-7}{ }^{0} \mathrm{C}^{-2}$, $\mathrm{C}=-4.22 \times 10^{-12}{ }^{0} \mathrm{C}^{-3}$. When the high order items are ignored, temperature change of $1{ }^{0} \mathrm{C}$ will approximately induce resistance variation of $0.38 \Omega$. Hence the specific temperature of the object can be retrieved by measuring resistance value of the Pt100. The commonly used temperature measuring modes to Pt100 include 2-wire ,3-wire and 4-wire methods. To reduce influence of linking wire on resistance measurement, our system adopts the 3-wire measuring method.

Using 3-wire resistance measurement ${ }^{[2]}$ based on constant voltage detection, we can effectively exclude the influence due to the wire resistance. The schematic diagram is shown as Fig. 2, where $R_{t}$ is the $\mathrm{Pt} 100$ thermal resistance, $\mathrm{r}$ is wire equivalent resistance, $\mathrm{Rv}$ is divider resistance, $\mathrm{VR}$ is standard reference voltage, $\mathrm{VAD}$ is reference voltage of the $\mathrm{ADC}, \beta$ is gain of the amplifier, and $\mathrm{I}$ is electric current running through the Pt100. 


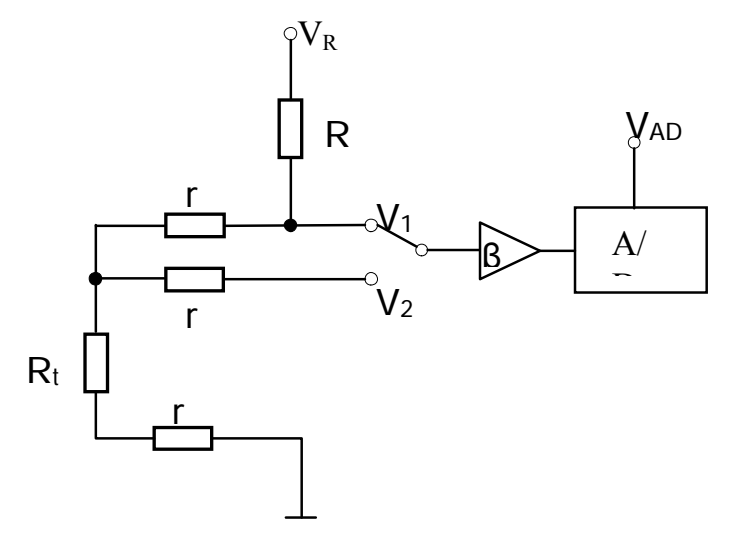

Fig. 2 Schematic diagram of 3-wire method to measure resistance of Pt100

Through Ohm's law and the virtual-short virtual-off feature, we can obtain the following relations:

$$
\begin{aligned}
& I=\frac{V_{R}}{R+2 r+R} . \\
& V_{1}=I\left(2 r+R_{i}\right) . \\
& V_{2}=I\left(r+R_{i}\right) .
\end{aligned}
$$

If $\mathrm{V}_{\mathrm{R}}, \mathrm{V}_{1}$ and $\mathrm{V}_{2}$ are known, $\mathrm{R}_{\mathrm{t}}$ can be reformed as Eq. 5:

$$
R=R_{v}\left(2 V_{2}-V_{1}\right) /\left(V_{R}-V_{1}\right)
$$

In this equation, $V_{1}$ and $V_{2}$ can be calculated by Eq. 6 and Eq. 7 :

$$
\begin{aligned}
& V_{1}=\frac{D_{1}}{\beta} V_{A D} . \\
& V_{2}=\frac{D_{2}}{\beta} V_{A D} .
\end{aligned}
$$

where $D_{1}$ and $D_{2}$ are $A D$ sampling value for measuring $V_{1}$ and $V_{2}$ respectively, $V_{A D}$ is reference voltage of the ADC. It can be seen that $V_{1}$ and $V_{2}$ depend on the value of $\beta . \beta$ and $R_{v}$ will vary due to circuit component characteristics, especially when those components are in batch production their performance can not keep exactly uniform, so we should consider errors due to $\beta$ and $R_{v}$ when designing specific circuit. In our system, we use precision resistor to calibrate values of $\beta$ and $R_{v}$, in addition replacing long wire by wire as short as possible (then the wire resistance turns to negligible). When $R_{t}$ is replaced by the precision resistance $R$, the relation equations will be

$$
V 1=V 2=\frac{R}{R+R} V_{R}=\frac{D}{\beta 2^{K}} V_{A D}
$$

We can use two precision resistors to get two measurements of $A D$ sampling value (obtaining $\mathrm{D}_{1}$ and $D_{2}$ ), then values of $\beta$ and $R_{v}$ can be retrieved by formulas: 


$$
\begin{aligned}
& R=\frac{D_{2}-D_{1}}{D_{1} R_{2}-D_{2} R_{1}} R_{1} R_{2} . \\
& \beta=\frac{R_{2}-R_{1}}{D_{1} R_{2}-D_{2} R_{1}} D_{1} D_{2} \frac{V_{A D}}{V_{R}} .
\end{aligned}
$$

To eliminate influence due to different reference sources, we make $V_{A D}$ and $V_{R}$ provided by the same reference source. The temperature collection circuit we designed is shown in Fig. 3. The measurement accuracy of the chosen adhesive PT100 is about $0.1^{\circ} \mathrm{C}$. AD7787 is chosen as ADC in our system, which chip has a built-in 24-bit low-noise $\Sigma$ - $\Delta$ type ADC, a differential input port and a buffered/unbuffered single-ended input port. AD7787 has its own internal clock, does not need user to provide external clock source, and is quite suitable for low-power low frequency measurements.

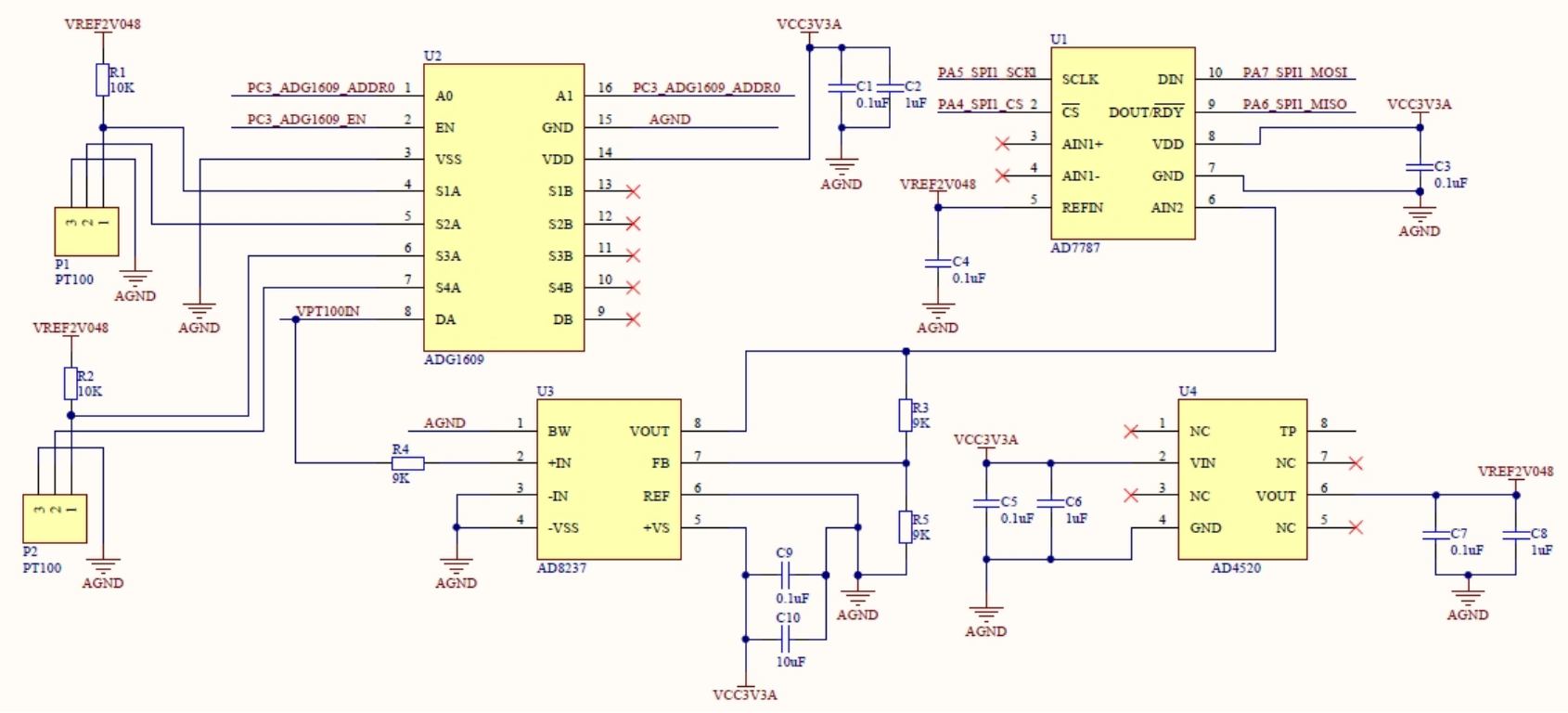

Fig. 3 The designed temperature collection circuit

In the system tests, we use a $100 \Omega$ and a $300 \Omega$ resistor both with five-ten thousand precision to resolve values of $\beta$ and $R_{v}$, and obtain $\beta=10.243386, R_{v}=10127.76469 \Omega$. We also measured on three resistors with fice-ten thousand precision $(150 \Omega, 200 \Omega, 250 \Omega$, respectively) by the proposed system, and result is $149.987 \Omega, 199.962 \Omega, 249.958 \Omega$, respectively. It can be obviously seen that measured resistance error is no more than $0.05 \Omega$. According to the rule that variation of temperature $1^{0} \mathrm{C}$ will approximately cause PT $100^{\prime}$ s resistance to change $0.38 \Omega$, we can deduce the measurement precision of our system is likely better than $0.2^{\circ} \mathrm{C}$.

\section{Circuit Design of the GPRS Wireless Transmission Module}

Our system applied a GPRS module developed by the Neoway corporation. For this module, nominal operating temperature range is $-40^{\circ} \mathrm{C} \sim 85^{\circ} \mathrm{C}$, EGSM900/DSC1800 dual frequency is available, signal reception sensitivity is better than $-107 \mathrm{dBm}$, maximum transmitting power is $2 \mathrm{~W}$, TCP/IP protocol is built-in, uplink/downlink signal transfer rate is up to $85.6 \mathrm{kbps}$, server mode and client mode are both configurable, standby current is $18 \mathrm{~mA}$, sleep current is less than $1 \mathrm{~mA}$, average power consumption is no more than 420mA at 4UP/1Down@Gamma=3 and no more than 190mA at 1UP/4Down@Gamma=3. Its good features make it a ideal choose for our system. 
Because of the maximum instantaneous current of this GPRS module is up to 2A, we chose a TPS54540 DC/DC module which can afford high conversion efficiency for 5A current. This DC/CD module has an enable output port, which can cut off power supply to the GPRS module if necessary, in addition it can reset the GPRS module when exceptions make GPRS crash down, so that it can effectively improve reliability of the whole system. The detailed power implement circuit of the GPRS module is shown in Fig. 4.

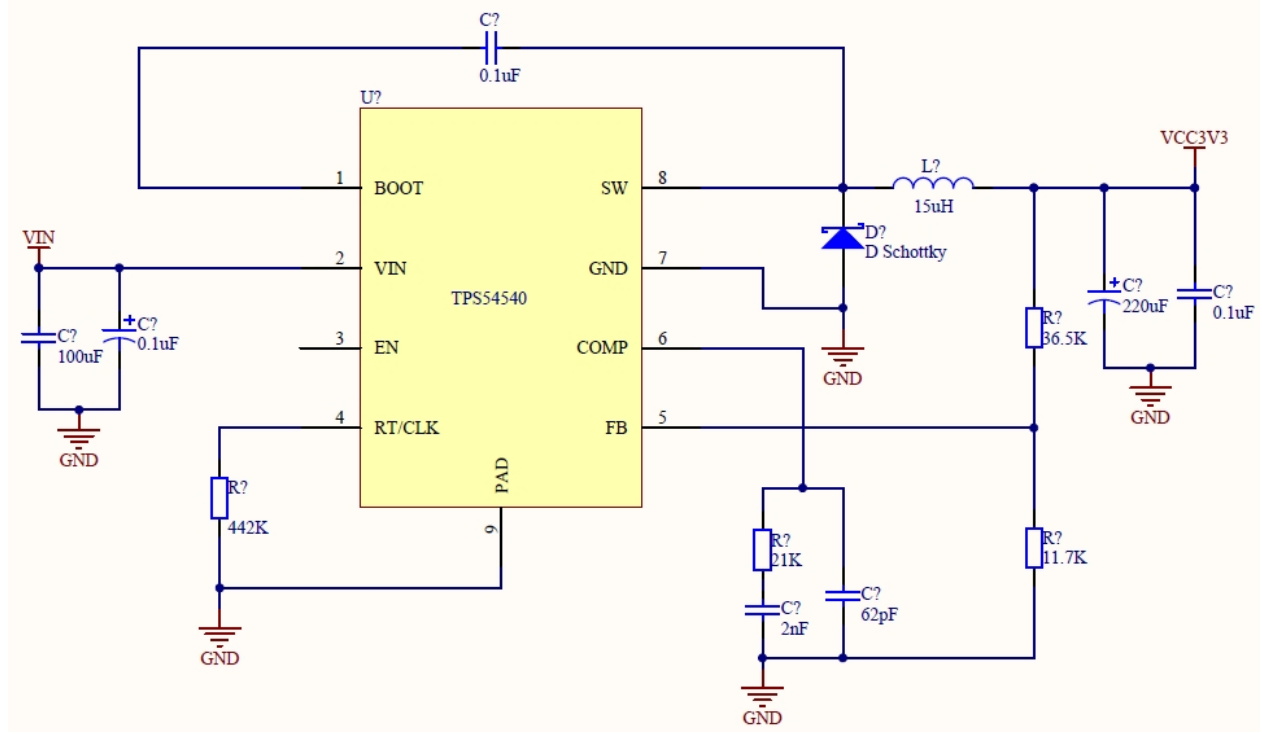

Fig.4 Power Circuit diagram of the GPRS module

In our system, the STM32 MCU will use AT instruction to control operation of GPRS module, so as to realize teletransmission of the measured data. Some important ATcommands ${ }^{[3][4]}$ are listed in Table 1 .

Table 1 Some important AT commands

\begin{tabular}{|c|c|}
\hline $\mathrm{AT}+\mathrm{CPIN} ?$ & SIM card detection \\
\hline $\mathrm{AT}+\mathrm{CCID}$ & Read the EF-CCID file on the SIM card \\
\hline $\mathrm{AT}+\mathrm{CSQ}$ & Determines the received signal strength indication \\
\hline AT+CREG? & Determines the registration status of the module \\
\hline $\mathrm{AT}+\mathrm{XISP}=0$ & Choose internal TCP/IP protocol stack \\
\hline AT+CGDCONT=“"”, “”, “” & Set APN \\
\hline $\mathrm{AT}+\mathrm{XGAUTH}=$ "“ ”, “"”, “”, “" ” & User authentication \\
\hline AT+CGATT? & Query GPRS attachment status \\
\hline $\mathrm{AT}+\mathrm{XIIC}=1$ & Make a PPP connection \\
\hline $\mathrm{AT}+\mathrm{XIIC} ?$ & Query PPP connection status contains the module's IP address \\
\hline AT+TCPSETUP=“"”, “”, “” & Establish a TCP connection \\
\hline AT+TCPSEND=“"”, “” & Send TCP/IP data \\
\hline +TCPRECV & Indicate receiving TCP/IP data \\
\hline
\end{tabular}

\section{Interface Design for Micro SD Card}

To realize local storage and backup of the data, we use a STM32 SDIO module to implement the interface to Micro SD card. Meanwhile, with the aid of open source FatFS file system source codes which is suitable for embedded development, we can conveniently realize i/o access of $\mathrm{FAT}^{[5]}$ format files. Only the low-level driver to SDIO is need to be coded, so development cycle can be significantly condensed. 


\section{Software Flow Chart}

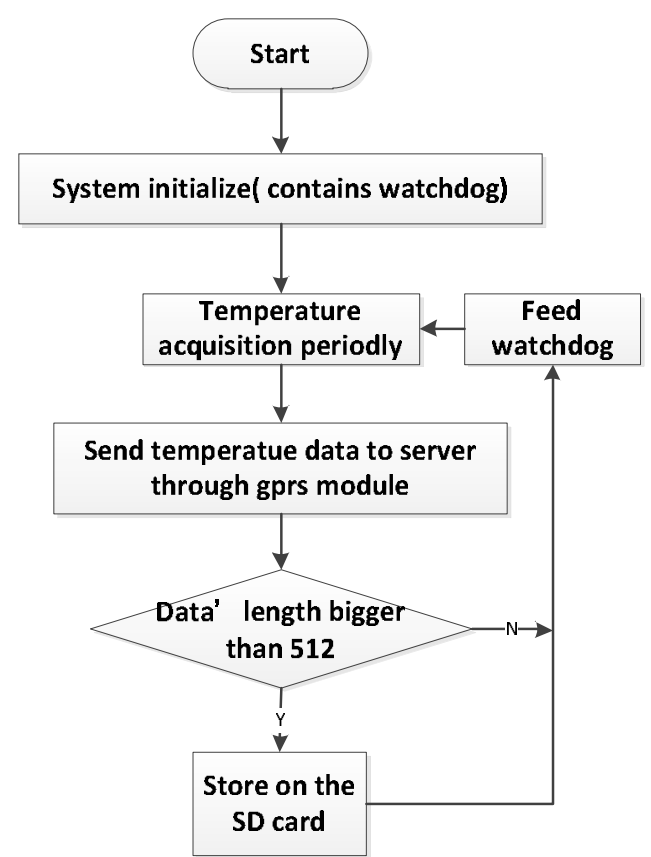

Fig. 5 Software flow chart

Flow chart of the software used for the proposed system is shown in Fig. 5. Firstly, when the system starts, the STM32 MCU will initialize components such as system clock, RS232, SPI, timer and watchdog, meanwhile make initialization settings for functional modules such as GPRS, OLED, ADC and Micro SD card. Once initialization finished, the MCU will trigger the timer to periodically collect temperature measurement data, deliver the data to remote server through GPRS module, then check the data size, if data size is no less than 512 bytes (one sector in SD card) , 512 bytes will be written onto the SD card (this operation can improve read/write speed of the SD card). After writing data to SD card, the MCU will reset the counter of the watchdog and wait for generation of the next collecting timer interrupt. Collection of temperature data mainly depends on measurement of Pt100's resistance. Based on Pt100 resistance, we can retrieve temperature value through look-at-table and linear fitting method. Another thing important is, to assure system reliability, the watchdog will inform the system to reset and restart in case of program exception.

\section{System Testing}

In system testing, the proposed system was used to measure temperature of a diffuse reflection gold panel and conduct real-time data transmission (see Fig.6). In the same time, a Fluke 51 II thermometer was monitoring temperature of the gold panel for comparison. We find the difference between measured values by these two methods is always within $\pm 0.10 \mathrm{C}$, indicating that temperature measured by our system is reliable enough to reach the expected goal. Moreover, considering municipal electricity is usually hard to access in field remote sensing experiment, our system intentionally uses low-power MCU and GPRS module capable of low-current sleep mode, so that the system can get power supply from lithium battery or solar cell panel, making it possible to perform long-term temperature measurement in unattended conditions. 


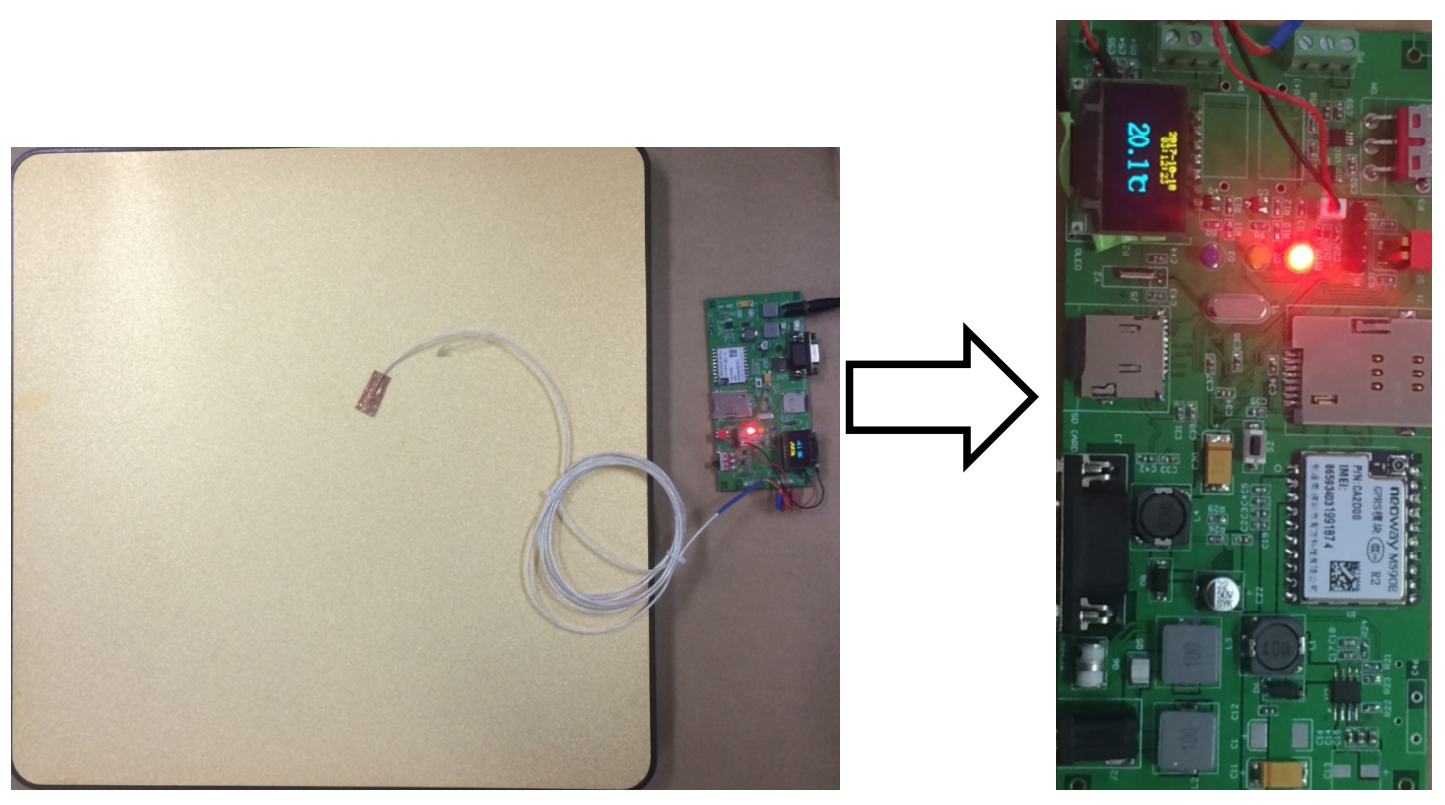

Fig.6 System test for measuring temperature of a diffuse reflection panel

\section{Conclusion and Future Work}

This paper describes the design and testing of a remote high precision temperature acquisition system based on GPRS. The system meets the requirement of unattended temperature measurement in field experiment or long-term field observation, is expected to be valuable for a variety of thermal remote sensing applications. In future work, several considerations will be investigated to further improve measurement precision of the system: (a) choosing high precision low drift resistor as $R_{v}$, so as to decrease error due to variation of $R_{v}$; (b) the output of power supply should have as low ripple wave as possible, and we should choose high PSRR (power supply rejection ratio) amplifier and ADC device, in order to reduce error from variation of power supply; (c) using temperature-controlled ${ }^{[6]}$ blackbody to calibrate performance of the system, to alleviate impact of Pt100 resistance bias on the temperature measurement results.

\section{Acknowledgements}

This work was financially supported by the National High Technology Research and Development Program of China under Grant(2014AA123201).

\section{References}

[1] STMicroelectronics: STM32F101xx, STM32F103xx, STM32F105xx and STM32F107xx advanced ARM®-based 32-bit MCUs

[2] GAN Ying-jun, ZHOU Hong-ping: Electronic Design Engineering Vol. 18 (2010),p. 31-33

[3] HU Bao-ling, FAN Han-bai and WANG Zhao Wei: Instrument Technique and Sensor No. 5, p. 50

[4] Neoway: Neo_M590E R2 GPRS module short message and tcp process introduction

[5] Information on http://elm-chan.org/fsw/ff/00index_e.html

[6] XU Jun, MENG Bing-huan, ZHAI Wen-chao, DING Lei and ZHENG Xiao-bing: Infrared and Laser Engineering No.8,p. 716-721 